\title{
Studies on the Effects of Saturated and Unsaturated Short-Chain Monocarboxylic Acids on the Energy Metabolism of Rat Liver Mitochondria
}

\author{
NIELS GREGERSEN \\ Research Laboratory for Metabolic Disorders, University Department of Clinical Chemistry, Aarhus \\ Kommunehospital, Aarhus, Denmark
}

\begin{abstract}
Summary
The effect of eight branched-chain amino acid metabolites, four metabolites from the $\beta$-oxidation, and the unphysiologic acid 4 pentenoic acid on the oxygen consumption rate of liver mitochondria oxidizing pyruvate, 2-oxo-glutarate, and L-palmitoylcarnitine has been investigated. The 12 metabolites are: propionic, isobutyric, 2-Me-butyric, isovaleric, acrylic, Me-acrylic, tiglic, Me-crotonic, butyric, hexanoic, crotonic, and 2-hexenoic acids. The oxidation rate of pyruvate was strongly inhibited by propionic, 4pentenoic, and isovaleric acids at $0.1,0.1$, and $1.0 \mathrm{mM}$, respectively. With 2-oxo-glutarate as substrate, the oxygen consumption rate was strongly inhibited at $0.1 \mathrm{mM}$ of propionic, 4-pentenoic, and isovaleric acids. The L-palmitoyl-carnitine oxidation rate was very strongly inhibited by $0.1 \mathrm{mM}$ 4-pentenoic acid, whereas butyric and hexanoic acids exerted a moderate inhibition at 0.1 $\mathrm{mM}$. Propionic acid inhibited L-palmitoylcarnitine oxidation slightly at $1.0 \mathrm{mM}$. It is argued that propionyl-CoA and isovalerylCoA inhibit pyruvate and 2-oxo-glutarate dehydrogenases directly, and the significance of the results for ketotic episodes in organic acidurias is discussed.
\end{abstract}

\section{Speculation}

The significant inhibition of energy metabolism by propionic and isovaleric acids compared to the other monocarboxylic acids suggests a direct inhibition of pyruvate and 2-oxo-glutarate dehydrogenases in mitochondria incubated with propionic or isovaleric acids. It may be speculated that the inhibition of the pyruvate metabolism in the intact cell, may cause an accumulation of pyruvate and lactate, and that the inhibition of the 2-oxo-glutarate oxidation may be ketogenetic by lowering the cellular oxaloacetate concentration. The excessive excretion and accumulation of propionic and/or isovaleric acid in acute episodes and the terminal phases of some organic acidurias is consistent with the hypothesis that these acids contribute to the pathophysiology of the acute disease states.

In children with inherited and acquired metabolic disturbances, short-chain monocarboxylic acids have been found in the blood and urine, especially in connection with acute crises. Large amounts of propionic acid are accumulated in patients with propionic acidemia and methylmalonic acidemia. and isovaleric acid is accumulated in patients with isovaleric acidemia $(13,18,24)$. Substantial amounts of propionic, isobutyric, 2-Me-butyric, isovaleric, butyric, and hexanoic acids have been detected in urine from a neonate with glutaric aciduria type II (19), and small amounts of the same acids, with the exception of butyric and hexanoic acids, were excreted (predominantly as conjugates (9)) during ketotic episodes in a patient with glutaryl-CoA dehydrogenase deficiency (glutaric aciduria). Elevated serum concentrations of propionic, isobutyric, isovaleric, butyric, and valeric acids have been found in patients suffering from Reye's syndrome (27, 28).

The acute crises and terminal phases of the mentioned disorders are often associated with coma. The ability of the straight shortchain monocarboxylic acids and of isovaleric acid to produce coma in experimental animals has been shown $(21,26)$, and it has been suggested that the coma seen in isovaleric acidemia and Rey's syndrome may be ascribed to these monocarboxylic acids $(26,28)$. Other features of the acute crises of the organic acidurias are lactic acidosis and, in most cases, ketosis $(9,13,18,19,24)$. These biochemical disturbances may be caused by disturbances of the energy metabolism associated with the citric acid cycle. As an approach to the question about the role of the monocarboxylic acids in these disturbances in patients with organic acidurias, the present study was undertaken. The effects of 13 metabolically interesting monocarboxylic acids on the oxidation rate of pyruvate, 2-oxo-glutarate, and $\mathrm{L}$-palmitoylcarnitine of isolated rat liver mitochondria are reported.

\section{MATERIALS AND METHODS}

Mitochondria were obtained from Wistar rats weighing 150 $200 \mathrm{~g}$. The rats were killed by a blow on the head followed by spinal dislocation. Within a min, the liver was removed, cut into small pieces, and chilled to $0-4^{\circ} \mathrm{C}$ in cold medium containing 300 $\mathrm{mM}$ sucrose, $5 \mathrm{mM}$ Hepes (30), and $1 \mathrm{mM}$ EDTA (31). The 1:5 liver/medium suspension was homogenized by means of a glass Potter Elverhjelm homogenizer with a loose piston (2 strokes). Centrifugation of the homogenate was performed at $4{ }^{\circ} \mathrm{C}$ and 400 $\mathrm{g}$ for $10 \mathrm{~min}$, the supernatant then being centrifuged at $8000 \mathrm{~g}$ for $10 \mathrm{~min}$. The mitochondrial pellet was washed 3 times before it was resuspended in medium to a concentration of $2-4 \mathrm{mg}$ protein/ $100 \mu \mathrm{l}$. Protein was measured by the method of Lowry et al. (16). Rate of oxygen consumption was measured polarographically by means of a Clark-type electrode (6) in a basic medium consisting of (final concentrations): $10 \mathrm{mM}$ sucrose, $100 \mathrm{mM} \mathrm{KCl}, 20 \mathrm{mM}$ Hepes, $5 \mathrm{mM} \mathrm{KH}_{2} \mathrm{PO}_{4}, 10 \mathrm{mM} \mathrm{MgCl}_{2}, 1 \%$ defatted serum albumin (5), and $2-3 \mathrm{mg}$ mitochondria in $3.3 \mathrm{ml}$ deionized and glass distilled water at $\mathrm{pH}=7.4$. Concentrations of substrates, monocarboxylic acids, malate, and ADP are given in the legends to the tables and figures. The designations state 3, for ADP-stimulated respiration, and state 4 , for ADP-limited respiration, are those used by Chance and Williams (3). Respiration control ratio ( $\mathrm{RCR}), \mathrm{ADP} / \mathrm{O}_{2}$-ratio $(\mathrm{P} / \mathrm{O})$, and oxygen consumption rate were measured according to Estabrook (6). 


\section{RESULTS}

The mitochondria used were always tightly coupled ( RCR > 5 ), and the measurements were performed within $3 \mathrm{hr}$ after the isolation. All experiments were performed with added albumin at a concentration of $1 \%(10)$. The inclusion of albumin caused the oxidation rate in state 3 to remain constant during the experiment, whereas the oxidation rate in this state diminished a little in the assays without albumin. This is probably due to a binding of the acids to the albumin, thus preventing a slight disruption of the mitochondria. A linear relationship between the state 3 oxygen consumption and the amounts of ADP added was found in the range $0.4-2.0 \mu$ mole. The linear relationship between oxygen consumption rate and amounts of mitochondrial protein was tested in all mitochondrial preparations.

Control assays, i.e., assays in which no short-chain monocarboxylic acid were added, were analyzed as every 4 th in the series. The mean of the oxygen consumption rate in the control assays (5-8) from each series was normalized to $100 \%$. The SEM for the substrates pyruvate, 2-oxo-glutarate, and L-palmitoylcarnitine was $2-3 \%$ in these control assays. The effect of each short-chain monocarboxylic acid was measured in at least two assays from different mitochondria preparations. Tables 1 and 2 show the results from the double determinations that were used in the calculation of the significant limits. From a precision of 11,11 , and $10 \%$, calculated from $s=\sqrt{\frac{d^{2}}{2 N}}$, the $99 \%$ significant limits can be calculated to be $80-120,80-120$, and $82-118 \%$ for pyruvate, 2-oxo-glutarate, and L-palmitoylcarnitine, respectively.

\section{EFFECTS ON PYRUVATE OXIDATION}

One series of measurements, showing the effect of isovaleric acid on the oxidation of pyruvate, is shown in Figure 1. States 3 and 4 oxidation rates were measured as the slopes of the curves. Calibration was performed with the basic medium saturated with air. The results in Table 1 show that the intermediates of fatty acid oxidation, hexanoic, and 2-hexenoic acids are stimulatory at the lower concentrations and inhibitory at the higher ones. The trend for butyric and crotonic acids is the same.

This picture is in contrast to the effects exerted by the inter- mediates of the branched-chain amino acid metabolism. Propionic and isovaleric acids are clearly inhibitory; propionic acid at all three concentrations tested, and isovaleric acid at 1.0 and $10 \mathrm{mM}$. The trend in the oxidation rate with added isobutyric, 2-Mebutyric, Me-acrylic, tiglic, and Me-crotonic is noteworthy. The inhibitory tendency at the lower concentrations seems to be counteracted by an induction of the oxidation rate at higher concentrations. 4-pentenoic acid is clearly inhibitory at all three concentration levels.

Table 2. Effects of saturated and unsaturated short-chain monocarboxylic acid on the oxygen consumption rate in state 3 of rat liver mitochondria oxidizing L-palmitoylcarnitine ${ }^{1}$

\begin{tabular}{lccc}
\hline & \multicolumn{3}{c}{ Rate of oxygen uptake $(\% \text { of control value })^{2}$} \\
\cline { 2 - 4 } Acid concentration & $0.1 \mathrm{mM}$ & $1.0 \mathrm{mM}$ & $10 \mathrm{mM}$ \\
\hline Propionic acid & $91(89-92)$ & $79(80-78)$ & $74(75-73)^{3}$ \\
Isobutyric acid & $92(95-89)$ & $92(92-92)$ & $97(88-105)$ \\
2-Me-butyric acid & $102(100-103)$ & $96(95-97)$ & $106(97-114)$ \\
Isovaleric acid & $96(95-96)$ & $93(99-87)$ & $97(93-100)$ \\
Butyric acid & $76(79-73)^{3}$ & $67(75-63)^{3}$ & $58(56-60)$ \\
Hexanoic acid & $78(73-83)^{3}$ & $77(75-78)^{3}$ & $41(29-52)^{3}$ \\
Acrylic acid & $96(94-97)$ & $99(91-107)$ & $63(74-56)^{3}$ \\
Me-acrylic acid & $107(100-113)$ & $98(98-97)$ & $81(80-81)^{3}$ \\
Tiglic acid & $101(87-115)$ & $104(93-115)$ & $96(80-111)$ \\
Me-crotonic acid & $95(96-94)$ & $92(89-94)$ & $92(89-94)$ \\
Crotonic acid & $97(100-93)$ & $99(98-100)$ & $67(67-67)^{3}$ \\
2-hexenoic acid & $101(93-109)$ & $91(82-100)$ & $71(52-89)^{3}$ \\
4-pentenoic acid & 14 & 14 & 11 \\
\hline
\end{tabular}

${ }^{1}$ Mitochondria (2-3 mg of protein) were preincubated at $30^{\circ} \mathrm{C}$ for 8 min in the basic medium (see Methods) containing malate $(2.5 \mathrm{mM})$ and acid $(0-10 \mathrm{mM})$. ADP $(2.0 \mu$ mole $)$ was then added and after $2 \mathrm{~min}$, the recording of state 3 for L-palmitoylcarnitine oxidation was started by the addition of L-palmitoylcarnitine $(0.03 \mathrm{mM})$. The figures in the Table represent the mean of two determinations, the results of which are shown in the brackets.

${ }^{2}$ The control value was defined as the mean of the 5-7 control assays conducted during each series (see Results).

${ }^{3}$ Mean values below the significant limit (see Results). The control rate of L-palmitoylcarnitine oxidation was $85-125 \mu \mathrm{atom} / \mathrm{min} / \mathrm{mg}$ protein.

Table 1. Effects of saturated and unsaturated short-chain monocarboxylic acids on the oxygen consumption rate in state 3 of liver mitochondria oxidizing pyruvate and 2-oxo-glutarate

\begin{tabular}{|c|c|c|c|c|c|c|}
\hline \multirow{3}{*}{$\frac{\text { Substrate }}{\text { Acid concentration }}$} & \multicolumn{6}{|c|}{ Rate of oxygen uptake (\% of control value $)^{2}$} \\
\hline & \multicolumn{3}{|c|}{ Pyruvate } & \multicolumn{3}{|c|}{ 2-oxo-glutarate } \\
\hline & $0.1 \mathrm{mM}$ & $1.0 \mathrm{mM}$ & $10 \mathrm{mM}$ & $0.1 \mathrm{mM}$ & $1.0 \mathrm{mM}$ & $10 \mathrm{mM}$ \\
\hline Propionic acid & $56(55-56)^{3}$ & $35(34-36)^{3}$ & $32(34-30)^{3}$ & $57(63-50)^{3}$ & $16(16-15)^{3}$ & $13(11-14)^{3}$ \\
\hline Isobutyric acid & $91(91-91)$ & $76(76-76)^{3}$ & $104(116-91)$ & $87(80-93)$ & $86(72-99)$ & $93(99-87)$ \\
\hline 2-Me-butyric acid & $87(89-85)$ & $72(79-64)^{3}$ & $89(93-85)$ & $102(111-92)$ & $90(96-84)$ & $83(85-81)$ \\
\hline Isovaleric acid & $104(115-93)$ & $52(49-54)^{3}$ & $40(41-38)^{3}$ & $75(65-89)^{3}$ & $22(20-24)^{3}$ & $35(36-33)^{3}$ \\
\hline Butyric acid & $111(125-96)$ & $103(115-91)$ & $95(108-81)$ & $96(75-116)$ & $100(84-114)$ & $101(88-114)$ \\
\hline Hexanoic acid & $125(125-125)$ & $117(125-108)$ & $69(64-74)^{3}$ & $105(95-115)$ & $100(90-109)$ & $81(70-91)$ \\
\hline Acrylic acid & $104(103-105)$ & $105(109-100)$ & $89(78-100)$ & $104(112-96)$ & $110(112-108)$ & $56(53-59)^{3}$ \\
\hline Me-acrylic acid & $84(68-100)$ & $88(75-100)$ & $94(83-105)$ & $102(99-102)$ & $98(99-96)$ & $95(93-96)$ \\
\hline Tiglic acid & $76(75-77)^{3}$ & $76(75-77)^{3}$ & $80(82-77)$ & $88(89-87)$ & $103(97-108)$ & $83(78-87)$ \\
\hline Me-crotonic acid & $81(89-72)$ & $81(82-80)$ & $101(100-101)$ & $85(80-90)$ & $85(89-81)$ & $92(91-93)$ \\
\hline Crotonic acid & $115(118-112)$ & $114(115-112)$ & $107(108-106)$ & $110(108-112)$ & $117(116-118)$ & $117(113-120)$ \\
\hline 2-hexenoic acid & $124(114-133)$ & $122(118-125)$ & $82(68-95)$ & $110(122-97)$ & $111(122-100)$ & $84(90-77)$ \\
\hline 4-pentenoic acid & $18^{3}$ & $14^{3}$ & $14^{3}$ & $41^{3}$ & $14^{3}$ & $28^{3}$ \\
\hline
\end{tabular}

Mitochondria (2-3 mg of protein) were preincubated at $30^{\circ} \mathrm{C}$ for $10 \mathrm{~min}$ in the basic medium (see Methods) containing substrate, i.e., pyruvate (10 $\mathrm{mM})$ /malate $(2.5 \mathrm{mM})$ or 2-oxo-glutarate $(10 \mathrm{mM})$, and acid $(0-10 \mathrm{mM})$. ADP $(1.6 \mu \mathrm{mole})$ was then added, and the oxygen consumption rate in states 3 and 4 recorded and expressed as the percent of those in the absence of added acid. The values for the two determinations on different mitochondria perparations are shown in brackets.

${ }^{2}$ The control value was defined as the mean of the 5-8 control assays conducted during each series (see Results)

${ }^{3}$ Mean values below the significant limit (see Results). The control rate of pyruvate and 2-oxo-glutarate oxidation was 67-100 and 101-152 $\mu$ atom/ $\mathrm{min} / \mathrm{mg}$ protein, respectively. 


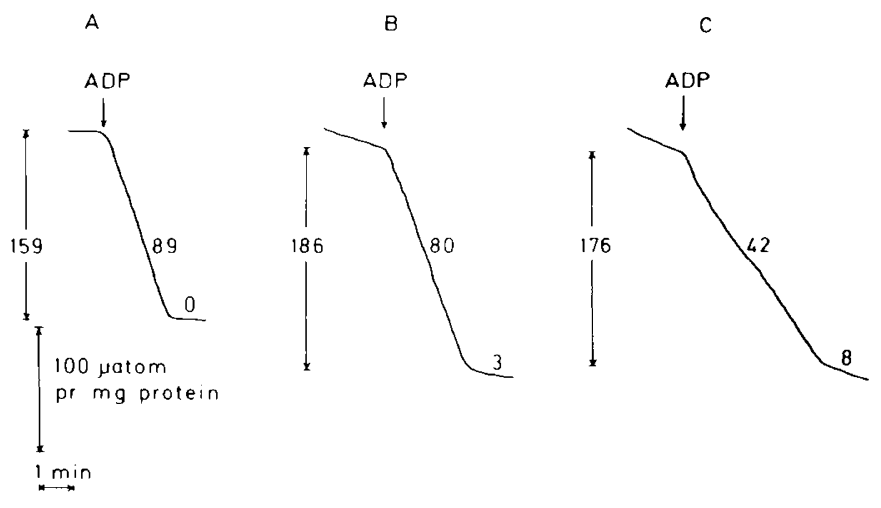

Fig. 1. Inhibition by isovaleric acid of the state 3 rate of oxidation of pyruvate in rat liver mitochondria. Mitochondria (3.3 $\mathrm{mg}$ of protein) were preincubated in basic medium containing pyruvate $(10 \mathrm{mM})$, malate $(2.5$ $\mathrm{mM})$, and isovaleric acid $(A: 0 \mathrm{mM}, B: 0.1 \mathrm{mM}$, and $C: 1.0 \mathrm{mM})$. After 10 $\mathrm{min}$ at $30^{\circ} \mathrm{C} \mathrm{ADP}(1.6 \mu$ mole) was added (indicated by an arrow) and the amounts of oxygen consumed in state 3 determined (ngatom/mg protein; indicated between vertical arrows). The oxygen consumption rates in states 3 and 4 (ngatom $0 / \mathrm{min} / \mathrm{mg}$ protein) are shown on the traces.

\section{EFFECT ON 2-OXO-GLUTARATE OXIDATION}

The results of the experiments in which the acid metalites were added to the assay systems oxidizing 2-oxo-glutarate are shown in Table 1 . The only statistically significant effects, with the exception of that of acrylic acid at $10 \mathrm{mM}$, are those exerted by propionic, isovaleric, and 4-pentenoic acids. They inhibit the oxygen consumption rate at all concentrations. The trend in the effects of the $\beta$-oxidation metabolites, hexanoic, and 2 -hexenoic acids is the same as that seen when pyruvate is used as substrate. There is no obvious trend in the effects of the five other branched-chain amino acid metabolites-isobutyric, 2-Me-butyric, Me-acrylic, tiglic, and Me-crotonic acids.

\section{EFFECT ON L-(-)-PALMITOYLCARNITINE OXIDATION}

The results of the experiments using L-palmitoylcarnitine as substrate are shown in Table 2 . The effects of the saturated fatty acid metabolites, butyric, and hexanoic acids, are significant at all three concentrations, whereas those of the unsaturated acids, crotonic, and 2-hexenoic acids, are only statistically significant at $10 \mathrm{mM}$. 4-pentenoic acid is very inhibitory at all concentrations, whereas the branched-chain amino acid metabolites exert effects that are either small or only seen at the highest concentration. However, the trend in the effect of propionic acid is very similar to that of the other straight-chain saturated acids, butyric, and hexanoic acids.

In the determination shown in Table 2 , the oxygen consumption may not originate exclusively from the $\beta$-oxidation, because the citric acid cycle was not blocked in these experiments. The slight but significant inhibition of propionic acid may, therefore, be explained by the inhibition of the 2-oxo-glutarate oxidation. However, blockage of succinate dehydrogenase with $20 \mathrm{mM}$ malonate did not eliminate the inhibition (results not shown).

\section{DISCUSSION}

The present investigation shows that propionic, isovaleric, and 4-pentenoic acids inhibit the oxidation rate of pyruvate and 2oxo-glutarate in rat liver mitochondria at concentrations $\leq 1 \mathrm{mM}$. The inhibitory effect of 4-pentenoic acid is known (22), but the mechanism has never been elucidated. Several mechanisms of inhibition by the acids are possible: 1) the acids may disrupt the mitochondrial membranes and thereby inhibit the oxidation process as has been proposed for the medium-chain monocarboxylic acids (22). This is unlikely, not only because the short-chain monocarboxylic acids are bad detergents, but also because the three inhibitory acids have physicochemical properties similar to those of the other noninhibitory acids tested. 2) The inhibition may be caused by a sequestration of free coenzyme-A in the mitochondria. Propionyl-CoA is accumulated rapidly in rat liver perfused with propionic acid (23), and a variety of short-chain monocarboxylic acids, among others, 2-Me-butyric acid, have been shown to form $\mathrm{CoA}$ derivatives in cell-free preparations of beef liver, at a rate similar to that of butyric acid (17). Butyric acid forms CoA derivatives rapid in liver preparation (29). As the pyruvate and 2-oxo-glutarate oxidation is CoA-dependant, the sequestration of CoA may contribute to the inhibition of the oxidation rate. This might be the explanation for the slight drop in the oxidation rates observed with nearly all the branched-chain acids, but it is improbable that the considerable inhibition exerted by propionic, isovaleric, and 4-pentenoic acids can be explained by this mechanism. 3) The acids or their CoA-derivatives may inhibit the pyruvate and 2-oxo-glutarate dehydrogenases directly. Bremer (2) has shown that propionyl-CoA inhibits purified pyruvate dehydrogenase competitively with respect to CoA. Therefore, it may be possible that isovaleryl-CoA and 4-pentenoyl-CoA also inhibit pyruvate dehydrogenase directly. This hypothesis is, at present, under investigation. As the 2-oxo-glutarate dehydrogenase complex resembles the pyruvate dehydrogenase complex (20), it is probable that the mechanism of inhibition of the two complexes by the acids are similar.

The very strong inhibition of L-palmitoylcarnitine oxidation by 4-pentenoic acid is in accordance with reports from other researchers $(7,12)$. Sequestration of free CoA probably contribute to this inhibition (7), but it has been shown that a metabolite of 4 pentenoic acid, 2,4-penta-dienoyl-CoA, inhibits the 3-oxo-acylCoA thiolase directly $(11,12)$. None of the other acids investigated in the present work inhibited the oxidation rate of L-palmitoylcarnitine as much as 4-pentenoic acid did, and it is, therefore, improbable that any of these acids exert an effect by the same mechanism as that of 4-pentenoic acid. However, propionic, butyric, and hexanoic acids are clearly inhibitory. The results with butyric and hexanoic acids are in agreement with earlier reported data (10). Because of the rapid mitochondrial activation of these acids to their CoA-derivatives (29), sequestration of CoA is likely to be the inhibitory mechanism. As propionyl-CoA is also accumulated in rat liver perfused with propionic acid (23), the same mechanism is likely to work in the assays with propionic acids. This slight inhibition of the $\beta$-oxidation by propionic acid is in contrast to the very strong inhibition found by Glasgow and Chase (8). However, the assay used by Glasgow and Chase produces ${ }^{14} \mathrm{CO}_{2}$ through the citric acid cycle from $1{ }^{14} \mathrm{C}$-palmitic acid. A decrease in ${ }^{14} \mathrm{CO}_{2}$ production by adding propionic acid may, therefore, also be caused by an inhibition of the citric acid cycle. It has been shown that propionate inhibits the oxidation of 2-oxoglutarate strongly. The same criticism may, therefore, be used against the experiments in Table 2. However, in a supplementary assay, in which malonate was added to block the citric acid cycle, the slight inhibition of the $\beta$-oxidation by propionic acid was reproduced. The "4-pentenoic acid effect" of propionic acid observed by Glasgow and Chase (8) is, therefore, most likely to be an inhibition of the citric acid cycle.

In biologic systems in which propionic and/or isovaleric acids and their CoA-derivatives are accumulated due to an enzyme deficiency or an inhibition of either propionyl-CoA carboxylase, or isovaleryl-CoA dehydrogenase, the inhibition of pyruvate dehydrogenase may cause an accumulation of pyruvate and, therefore, of lactate. The inhibition of 2-oxo-glutarate dehydrogenase, which is a citric acid cycle enzyme, must result in reduced citric acid cycle activity. The energy production may thus be reduced and the concentration of oxaloacetate, necessary for citrate formation, may be lowered. If fatty acids are metabolized, this low oxaloacetate concentration may promote ketone body formation $(14,15)$.

The inhibition of the energy production through the citric acid 
cycle by isovaleric acid and not by tiglic and 3-Me-crotonic acid parallels the results of Teychenne et al. (26), who found that isovaleric acid increased the slow-wave electrical activity in rabbit brain, whereas tiglic and 3-Me-crotonic acids showed much less activity.

The accumulated isovaleric acid or more probably isovalerylCoA may thus, besides explaining the coma (26), also contribute to the lactic acidosis and ketosis of isovaleric acidemia and glutaric aciduria type II, the latter of which is also characterized by a large excretion of isovaleric acid (19). The ketosis in glutaric aciduria type II was not very pronounced, but other urinary constituents, i.e., ethylmalonic acid and $\mathrm{C}_{6}-\mathrm{C}_{10}$-dicarboxylic acids (19), indicate an inhibited $\beta$-oxidation, which may prevent the ketogenesis. In patients with propionic acidemia, substantial amounts of methylcitric acid have been found in the urine (1). This compound has been shown to inhibit several enzymes of the citric acid cycle (4), and may therefore, together with the propionic acid (propionyl$\mathrm{CoA}$ ), contribute to the ketogenesis by lowering the oxaloacetate concentration

In the other conditions, i.e., glutaryl-CoA dehydrogenase deficiency (9) and Reye's syndrome $(27,28)$, in which short-chain monocarboxylic acids have been detected in blood or urine, the accumulation of the CoA-derivative of the toxic acids seems to be much lower than in propionic, isovaleric, and methylmalonic acidemias and in glutaric aciduria type II. This could mean that the contribution to the biochemical and clinical features of the accumulated acyl-CoA are less than in the acidurias/emias just mentioned. The argument has been put forward (25), that the serum concentrations of monocarboxylic acids in Reye's syndrome are far too low to exert any toxic effects. However, if the accumulated acids are produced inside the mitochondrion, in which they exert the effects, knowledge about the serum concentration is of very little value in predicting the concentration of the acids at their sites of action.

\section{REFERENCES AND NOTES}

1. Ando, T., Rasmussen, K., Wright, J. M., and Nyhan, W. L.: Isolation and identification of methylcitrate, a major metabolic product of propionate in patients with propionic acidemia. J. Biol. Chem., 247: 2200 (1972).

2. Bremer, J.: Pyruvate dehydrogenase, substrate specificity and product inhibition. Eur. J. Biochem., 8: 535 (1969).

3. Chance, B., and Williams, G. R.: Respiratory enzymes in oxidative phosphorylation. I. Kinetics of oxygen utilization. J. Biol. Chem., 217: 383 (1955).

4. Cheema-Dhadli, S., Leznoff, C. C., and Halperin, M. L.: Effect of 2-methylcitrate on citrate metabolism: implications for the management of patients with propionic acidemia and methylmalonic aciduria. Pediatr. Res., 9: 905 (1975)

5. Chen, R. F.: Removal of fatty acids from serum albumin by charcoal treatment. J. Biol. Chem., 242: 173 (1967).

6. Estabrook. R. W: Mitochondrial respiration control and the polarographic measurement of ADP: 0 ratios. In: S. P. Colowick and N. O. Kaplan: Methods in Enzymology. Vol. 10, p. 41. (Academic Press, New York, 1967).

7. Fukami, M. H., and Williamson, J. R.: On the mechanism of inhibition of fatty acid oxidation by 4-pentenoic acid in rat liver mitochondria. J. Biol. Chem., 246: $1206(1971)$
8. Glasgow, A. M., and Chase, H. P.: Effects of propionic acid on fatty acid oxidation and ureagenesis. Pediatr. Res., 10:683 (1976).

9. Gregersen, N., and Brandt, N. J.: Ketotic episodes in glutaryl-CoA dehydrogenase deficiency (glutaric aciduria). Pediatr. Res., 13: 977 (1979).

10. Hird, F. J. R., and Weidemann, M. J.: Oxidative phosphorylation accompanying oxidation of short-chain fatty acids by rat liver mitochondria. Biochem. J., 98 378 (1966).

11. Holland, P. C., Senior, A. E., and Sherratt, H. S. A.: Biochemical effects of the hypoglycaemic compound pent-4-enoic acid and related non-hypoglycaemic fatty acid; effects of their CoA-esters on enzymes of fatty acid oxidation. Biochem. J., 136: 173 (1973).

12. Holland, P. C., and Sherratt, H. S. A.: Biochemical effects of the hypoglycaemic compound pent-4-enoic acid and related non-hypoglycaemic fatty acids; effects of the free acids and their carnitine esters on COA-dependent oxidation in rat liver mitochondria. Biochem. J., 136: 157 (1973)

13. Hommes, F. A. and van den Berg, C. J.: Inborn errors of metabolism. (Academic Press, London, 1973).

14. Krebs, H. A.: The regulation of the release of ketone bodies by the liver. In: G. Weber: Advances in Enzyme Regulation. Vol. 4, p. 339 (Pergamon Press, Oxford, 1966).

15. Lopes-Cardozo, M., and Berg, van den, S. G.: Ketogenesis in isolated rat liver mitochondria, relationships with the citric acid cycle and with the mitochondrial energy state. Biochim. Biophys. Acta, 283: 1 (1972).

16. Lowry, O. H., Rosebrough, N. J., Farr, A. L., and Randall, R. J.: Protein measurement with the folinphenol reagent. J. Biol. Chem., 193: 265 (1951).

17. Mahler, H. R., and Wakil, S. J.: Studies on fatty acid oxidation. I. Inzymatic activation of fatty acids. J. Biol. Chem., 204: 453 (1953).

18. Nyhan, W. L.: Heritable disorders of amino acid metabolism, pattern of clinical expression and genetic variation. (John Wiley and Sons, New York, 1974)

19. Przyrembel, H., Wendet, U., Becker, J., Bremer, H. J., Bruinvis, L., Ketting, D., and Wadman, S. K.: Glutaric aciduria type II; report on a previously undescribed metabolic disorder. Clin. Chim. Acta, 66: 227 (1976).

20. Reed, L. J. and Cox, D.: Multienzyme complexes. In: P. D. Boyer: The Enzymes. p. 213 (Academic Press, New York, 1970).

21. Samson, F. E., Dahl, N., and Dahl, D. R.: A study on the narcotic action of short-chain fatty acids. J. Clin. Invest., 35: 1291 (1956).

22. Senior, A. E. and Sherratt, H. S. A.: Biochemical effects of the hypoglycaemic compound pent-4-enoic acid and related non-hypoglycaemic fatty acids; oxidative phosphorylation and mitochondrial oxidation of pyruvate, 3-OH-butyrate and tricarboxylic acid-cycle intermediates. Biochem. J., 110: 499 (1968).

23. Søling, H. D.: Anion transport through the inner metochondrial membrane-site of regulation of gluconeogenesis? In: F. Lundquist and N. Tygstrup: Regulation of Hepatic Metabolism. p. 48 (Munksgaard, Copenhagen, 1974).

24. Tanaka, K.: Disorders of organic acid metabolism. In: G. E. Gaul: Biology of Brain Function. Vol. 3, p. 145. (Plenum Press, New York, 1975).

25. Tanaka, K.: Jamaican vomiting sickness and Reye's syndrome. N. Engl. J. Med., 295: 1481 (1977).

26. Teychenne, P. F., Walters, I., Claveria, L. E., Calne, D. B., Price, J., MacGillivary, B. B. and Gompertz, D.: The encephalopathic action of five-carbon-atom fatty acids in the rabbit. Clin. Sci. Mol. Med., 50: 463 (1976).

27. Trauner, D. A., Nyhan, W. L., and Sweetman, L.: Jamaican vomiting sickness and Reye's syndrome. N. Engl. J. Med., 295: 1481 (1977).

28. Trauner, D. A., Nyhan, W. L., and Sweetman, L.: Short-chain organic acidemia and Reye's syndrome. Neurology, 25: 296 (1975).

29. Williamson, D. H., Ellington, E. V., Ilic, V., and Saal, J.: Hepatic effects of saturated and unsaturated short-chain fatty acids and the control of ketogenesis. In: F. Lundquist and N. Tygstrup: Regulation of Hepatic Metabolism. p. 191 (Munksgaard, Copenhagen, 1974).

30. Hepes: $\mathrm{N}$-2-hydroxyethylpiperazine- $\mathrm{N}^{\prime}$-2-ethanesulfonic acid.

31. EDTA: Ethylenedinitrilotetra acetic acid.

32. This research was supported by the Danish Medical Research Council.

33. Requests for reprints should be addressed to: Niels Gregersen. Department of Clinical Chemistry, Aarhus Kommunehospital, DK-800 Aarhus C, Denmark.

34. Received for publication May 22, 1978.

35. Accepted for publication November 17, 1978 